INPLASY

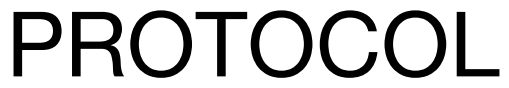

To cite: Liu et al. Accuracy of ultrasonic artificial intelligence in diagnosing benign and malignant breast diseases: $A$ protocol for systematic review and meta-analysis. Inplasy protocol 2021110087. doi: 10.37766/inplasy2021.11.0087

Received: 24 November 2021

Published: 24 November 2021

Corresponding author:

Qiyu Liu

863271992@qq.com

Author Affiliation:

Ultrasound department of the

First Affiliated Hospital of

Dalian Medical University.

Support: Liaoning Natural

Science Found.

Review Stage at time of this submission: Completed but not published.

Conflicts of interest:

None declared.

\section{Accuracy of ultrasonic artificial intelligence in diagnosing benign and malignant breast diseases: A protocol for systematic review and meta-analysis}

Liu, QY1; Qu, MJ2; Sun, LP3; Wang, $\mathrm{H}^{4}$.

Review question / Objective: Accuracy of ultrasonic artificial intelligence in diagnosing benign and malignant breast diseases. Methodological quality was independently assessed by 2 researchers based on the quality assessment of studies of diagnostic accuracy studies (QUADAS) tool. The QUADAS criteria included 14 assessment items. Each of these items was scored as "yes" (2), "no" (0), or "unclear"(1). The QUADAS score ranged from 0 to 28 , and a score $\geq 22$ indicated good quality. Any disagreements between 2 investigators will be solved through discussion or consultation by a $3 \mathrm{rd}$ investigator.

Condition being studied: Benign and malignant breast diseases. Two authors will independently select the trials according to the inclusion criteria, and import into Endnote $X 9$. Then remove duplicated or ineligible studies. Screen the titles, abstracts, and full texts of all literature to identify eligible studies. All essential data will be extracted using previously created data collection sheet by 2 independent authors.

INPLASY registration number: This protocol was registered with the International Platform of Registered Systematic Review and Meta-Analysis Protocols (INPLASY) on 24 November 2021 and was last updated on 24 November 2021 (registration number INPLASY2021110087).

\section{INTRODUCTION}

Review question / Objective: Accuracy of ultrasonic artificial intelligence in diagnosing benign and malignant breast diseases. Methodological quality was independently assessed by 2 researchers based on the quality assessment of studies of diagnostic accuracy studies (QUADAS) tool. The QUADAS criteria included 14 assessment items. Each of these items was scored as "yes" (2), "no" (0), or "unclear"(1). The QUADAS score ranged from 0 to 28, and a score $\geq 22$ indicated good quality. Any 
disagreements between 2 investigators will be solved through discussion or consultation by a 3rd investigator.

Condition being studied: Benign and malignant breast diseases. Two authors will independently select the trials according to the inclusion criteria, and import into Endnote X9. Then remove duplicated or ineligible studies. Screen the titles, abstracts, and full texts of all literature to identify eligible studies. All essential data will be extracted using previously created data collection sheet by 2 independent authors.

\section{METHODS}

Participant or population: The patients should be those who had undergone breast diseases

Intervention: This study compare Al with pathology for diagnosing breast diseases.

Comparator: This study compare Al with pathology for diagnosing breast diseases.

Study designs to be included: This study was conducted in accordance with the PRISMA (Preferred Reporting Items for Systematic Reviews and Meta-Analyses) guidelines.

Eligibility criteria: Type of study. This study will only include high quality clinical cohort or case control studies. Type of patients. The patients should be those who had undergone breast diseases. Intervention and comparison. This study compare Al with pathology for diagnosing breast diseases.Type of outcomes. The primary outcomes include sensitivity, specificity, positive and negative likelihood ratio, diagnostic odds ratio, and the area under the curve of the summary receiver operating characteristic.

Information sources: PubMed, Web of Science, Cochrane Library, and Chinese biomedical databases will be searched from their inceptions to the October 31, 2021, without language restrictions.
Main outcome(s): Its findings will provide helpful evidence for the accuracy of $\mathrm{Al}$ in the differential diagnosis of benign and malignant breast diseases.

Quality assessment / Risk of bias analysis: Two authors will independently select the trials according to the inclusion criteria, and import into Endnote X9. Then remove duplicated or ineligible studies. Screen the titles, abstracts, and full texts of all literature to identify eligible studies. All essential data will be extracted using previously created data collection sheet by 2 independent authors. Discrepancies in data collection between 2 authors will be settled down through discussion with the help of another author. The following data will be extracted from each included research: the first authors surname, publication year, language of publication, study design, sample size, number of lesions, source of the subjects, instrument, "gold standard," and diagnostic accuracy. The true positives, true negatives, false positives, and false negatives in the fourfold $(2 \times 2)$ tables were also collected. Methodological quality was independently assessed by 2 researchers based on the quality assessment of studies of diagnostic accuracy studies (QUADAS) tool.The QUADAS criteria included 14 assessment items. Each of these items was scored as "yes" (2), "no" (0), or "unclear"(1). The QUADAS score ranged from 0 to 28 , and a score $\geq 22$ indicated good quality. Any disagreements between 2 investigators will be solved through discussion or consultation by a 3rd investigator.

Strategy of data synthesis: The STATA version 14.0 (Stata Corp, College Station, TX, USA) and Meta-Disc version 1.4 (Universidad Complutense, Madrid, Spain) softwares were used for meta-analysis.

Subgroup analysis: We also performed sub group and meta-regression analyses to investigate potential sources of heterogeneity. To evaluate the influence of single studies on the overall estimate, a sensitivity analysis was performed. We conducted Begg's funnel plots and Egger's 
linear regression tests to investigate publication bias.

Sensitivity analysis: We calculated the pooled summary statistics for sensitivity, specificity, positive and negative likelihood ratio, and diagnostic odds ratio with their $95 \%$ confidence intervals. The summary receiver operating characteristic curve and corresponding area under the curve were obtained. The threshold effect was assessed using Spearman correlation coefficients. The Cochran's Q-statistic and I test were used to evaluate potential heterogeneity between studies. If significant heterogeneity was detected(Q test $\mathrm{P} 50 \%$ ), a random effects model or fixed effects model was used.

Country(ies) involved: China.

Keywords: artificial intelligence, metaanalysis, breast diseases.

Contributions of each author:

Author 1 - qiyu Liu.

Email: 863271992@qq.com

Author 2 - meijing Qu.

Email: 1439031356@qq.com

Author 3 - lipeng Sun.

Email: lipeng Sun

Author 4 - hui Wang.

Email: Indlnsd@163.com 